\title{
HANKEL PFAFFIANS, DISCRIMINANTS AND KAZHDAN-LUSZTIG BASES
}

\author{
Alain Lascoux
}

\begin{abstract}
We use Kazhdan-Lusztig bases of representations of the symmetric group to express Pfaffians with entries $\left(a_{i}-a_{j}\right) h_{i+j}$. In the case where the parameters $a_{i}$ are specialized to successive powers of $q$, and the $h_{i}$ are complete functions, we obtain the $q$-discriminant.
\end{abstract}

Hankel matrices are matrices constant along anti-diagonals. A prototype is $M=\left|h_{i+j}\right|_{i, j=1, \ldots, n}$, with indeterminates $h_{i}$ in a commutative ring.

With one more set of indeterminates $a_{i}$, and an integer $k \in \mathbb{Z}$, one defines the Hankel Pfaffian $\mathfrak{P f}(\mathbf{a}, \mathbf{h}, n, k)$ to be the Pfaffian of the antisymmetric matrix $\mathfrak{M}(\mathbf{a}, \mathbf{h}, n, k)$ of order $2 n$ with entries $\left(a_{i}-a_{j}\right) h_{i+j-3+k}$. This is the Pfaffian that we shall study in this text. Such Pfaffians with $a_{i}=i$ or $a_{i}=q^{i}$ and special $h_{i}$ have been considered by Ishikawa, Tagawa, Zeng [5].

Hankel matrices, when the $h_{i}$ are identified with complete functions of an alphabet of cardinality $n$, are related to resultants, Bezoutians, orthogonal polynomials, continued fractions, etc [10]. We show similarly in Section 2 and Section 5 that Hankel Pfaffians in complete functions allow to express resultants, Bezoutians, $q$-discriminants, and give several determinantal expressions of such Pfaffians.

The Hankel Pfaffian $\mathfrak{P f}(\mathbf{a}, \mathbf{h}, n, k)$ can be studied by mere algebraic manipulations, this is what we do in Section 2. However, it is much more fruitful to use the action of the symmetric group on the indeterminates $a_{i}$. In [11], we have shown how to diagonalize Pfaffians using Young's idempotents. In the present case, it is more convenient to use the bases of Kazhdan and Lusztig [7]. Theorem 13 shows, indeed, that $\mathfrak{P f}(\mathbf{a}, \mathbf{h}, n, k)$ is diagonal in a pair of adjoint Kazhdan-Lusztig bases.

Apart from the theory of symmetric functions, we shall need properties of representations of the symmetric group, that we recall in Section 3. Since the

Received April 6, 2011.

2010 Mathematics Subject Classification. 05E05, 05E10, 15A15, $20 \mathrm{C} 30$.

Key words and phrases. Pfaffians, discriminants, Kazhdan-Lusztig bases.

This article based on a talk given at the First Euro-Korean Conference on Groups and Related topics, March 2011, Pohang University of Science and Technology(POSTECH). 
combinatorics of Kazhdan and Lusztig bases are not well known, we give in this section more properties than is needed proper for the computation of Pfaffians.

\section{Symmetric functions}

We recall some properties of symmetric functions, following the conventions of [10] rather than more classical ones as found in the book of I. G. Macdonald.

\subsection{Schur functions}

Given a sequence $h_{0}=1, h_{1}, \ldots$, given an integer $n$ and $u, v \in \mathbb{N}^{n}$, one defines the Schur function $S_{v}$ to be the determinant of $\left[h_{v_{j}+j-i}\right]_{i, j=1, \ldots, n}$, and the skew Schur function $S_{v / u}$ to be the determinant of $\left[h_{v_{j}+j-i-u_{i}}\right]_{i, j=1, \ldots, n}$, putting $h_{i}=0$ for $i<0$ (but this convention will be changed later).

Given two finite alphabets $\mathbf{x}=\left\{x_{1}, \ldots, x_{n}\right\}$ and $\mathbf{y}=\left\{y_{1}, \ldots, y_{m}\right\}$, the complete functions $h_{i}(\mathbf{x}-\mathbf{y})$ of $\mathbf{x}-\mathbf{y}$ are defined by the generating series

$$
\prod_{i=1, \ldots, m}\left(1-z y_{i}\right) \prod_{i=1, \ldots, n}\left(1-z x_{i}\right)^{-1}=\sum_{0}^{\infty} z^{i} h_{i}(\mathbf{x}-\mathbf{y}) .
$$

Determinants of order $n$ in the complete functions of $\mathbf{x}$ satisfy [10, Th.1.8.3]

$$
\operatorname{det}\left(h_{v_{j}+j-i+u_{n-i+1}}(\mathbf{x})\right)=S_{v}(\mathbf{x}) S_{u}(\mathbf{x}), u, v \in \mathbb{N}^{n} .
$$

Similarly, for $r \geq 0, u, v \in \mathbb{N}^{n}$ such that $u \leq r^{n}$, one has

$$
S_{\left(v+r^{n}\right) / u}(\mathbf{x})=S_{v}(\mathbf{x}) S_{r-u_{n}, \ldots, r-u_{1}}(\mathbf{x}) .
$$

Schur functions of a difference of alphabets factorize, when the components of $v$ are big enough [10, Prop.1.4.3]

$$
S_{v+m^{n}}(\mathbf{x}-\mathbf{y})=\prod_{i=1, \ldots, n} \prod_{j=1, \ldots, m}\left(x_{i}-y_{j}\right) S_{v}(\mathbf{x}), v \in \mathbb{N}^{n} .
$$

\subsection{Invariance by translation of indices}

Given $\mathbf{x}$ of cardinality $n$, the sequence $h_{i}(\mathbf{x})$ is a recurrent sequence

$$
\sum_{i=0}^{n}(-1)^{i} e_{i}(\mathbf{x}) h_{k-i}(\mathbf{x})=0 \quad \text { for } k \geq n
$$

that one can extend, following Wronski, into a recurrent sequence $\left\{h_{k}(\mathbf{x}): k \in\right.$ $\mathbb{Z}\}$ by requiring relation (4) for all $k \in \mathbb{Z}$ and imposing the initial conditions $h_{-1}(\mathbf{x})=0=\cdots=h_{1-n}(\mathbf{x})[6]$.

From now on, the notation $h_{k}(\mathbf{x})$, as well as the different determinants in the $h_{k}(\mathbf{x})$, will use this convention. For example, a skew Schur function is defined for any pair $v, u \in \mathbb{Z}^{n}: S_{v / u}(\mathbf{x})=\operatorname{det}\left(h_{v_{j}-u_{i}+j-i}(\mathbf{x})\right)$. In fact, one has

$$
\frac{1}{x_{1} \cdots x_{n}} S_{v / u}(\mathbf{x})=S_{\left(v-1^{n}\right) / u}(\mathbf{x})=S_{v /\left(u+1^{n}\right)}(\mathbf{x}),
$$

so that, up to powers of $x_{1} \cdots x_{n}$, one can recover indices in $\mathbb{N}^{n}$. 
The properties of determinants of order $n$ in the $h_{i}(\mathbf{x})$ extend without further ado. For any $u, v \in \mathbb{Z}^{n}$, any $r \in \mathbb{Z}^{n}$, one has

$$
S_{v / u}(\mathbf{x})=S_{v+r^{n}}(\mathbf{x}) S_{r-u_{n}, \ldots, r-u_{1}}(\mathbf{x}) .
$$

For example, for $n=3$, one has $h_{-1}(\mathbf{x})=0, h_{-2}(\mathbf{x})=0, h_{-3}(\mathbf{x})=\left(x_{1} x_{2} x_{3}\right)^{-1}$ and

$$
S_{023 / 001}(\mathbf{x})=\left|\begin{array}{ccc}
h_{0}(\mathbf{x}) & h_{3}(\mathbf{x}) & h_{5}(\mathbf{x}) \\
h_{-1}(\mathbf{x}) & h_{2}(\mathbf{x}) & h_{4}(\mathbf{x}) \\
h_{-3}(\mathbf{x}) & h_{0}(\mathbf{x}) & h_{2}(\mathbf{x})
\end{array}\right|
$$

factorizes into $\left(x_{1}^{-1}+x_{2}^{-1}+x_{3}^{-1}\right) S_{023}(\mathbf{x})$, but this is not the case of the determinant $\left|\begin{array}{ccc}h_{0}(\mathbf{x}) & h_{3}(\mathbf{x}) & h_{5}(\mathbf{x}) \\ 0 & h_{2}(\mathbf{x}) & h_{4}(\mathbf{x}) \\ 0 & h_{0}(\mathbf{x}) & h_{2}(\mathbf{x})\end{array}\right|$ corresponding to the conventions $h_{k}(\mathbf{x})=0$ for $k<0$.

More generally, given any alphabet $\mathbf{y}=\left\{y_{1}, \ldots, y_{m}\right\}$, then $h_{i}(\mathbf{x}-\mathbf{y})$ is a recursive sequence satisfying the same recursion (4), and therefore can be extended to negative indices. The corresponding skew Schur functions still satisfy, for $u, v \in \mathbb{Z}^{n}$,

$$
\frac{1}{x_{1} \cdots x_{n}} S_{v / u}(\mathbf{x})=S_{\left(v-1^{n}\right) / u}(\mathbf{x})=S_{v /\left(u+1^{n}\right)}(\mathbf{x}) .
$$

For example, for $n=2=m$, one has $h_{1}(\mathbf{x}-\mathbf{y})=x_{1}+x_{2}-y_{1}-y_{2}, h_{0}(\mathbf{x}-\mathbf{y})=$ $1-y_{1} y_{2}\left(x_{1} x_{2}\right)^{-1}, h_{-1}(\mathbf{x}-\mathbf{y})=\left(y_{1}+y_{2}\right)\left(x_{1} x_{2}\right)^{-1}-y_{1} y_{2}\left(x_{1}+x_{2}\right)\left(x_{1} x_{2}\right)^{-2}$ and

$$
\begin{aligned}
S_{02 / 00}(\mathbf{x}-\mathbf{y}) & =\left|\begin{array}{cc}
h_{0}(\mathbf{x}-\mathbf{y}) & h_{3}(\mathbf{x}-\mathbf{y}) \\
h_{-1}(\mathbf{x}-\mathbf{y}) & h_{2}(\mathbf{x}-\mathbf{y})
\end{array}\right|=\left|\begin{array}{cc}
1-e_{2}^{y} e_{2}^{-1} & h_{3}-e_{1}^{y} h_{2}+e_{2}^{y} h_{1} \\
e_{1}^{y} e_{2}^{-1}-e_{2}^{y} e_{1} e_{2}^{-2} & h_{2}-e_{1}^{y} h_{1}+e_{2}^{y}
\end{array}\right| \\
& =\left(x_{1} x_{2}\right)^{-2} S_{24 / 00}(\mathbf{x}-\mathbf{y})=\left(x_{1} x_{2}\right)^{-2} R(\mathbf{x}, \mathbf{y})\left(x_{1}^{2}+x_{1} x_{2}+x_{2}^{2}\right),
\end{aligned}
$$

writing $e_{i}, h_{i}$ for the functions of $\mathbf{x}$, and $e_{1}^{y}, e_{2}^{y}$ for those of $\mathbf{y}$.

\subsection{Bezoutians and resultants}

Given $\mathbf{x}=\left\{x_{1}, \ldots, x_{n}\right\}$, the remainder of a polynomial $f(y)$ modulo $S_{n}(y-\mathbf{x})$ is the only polynomial $\mathcal{R}_{\mathbf{x}} f$ of degree $\leq n-1$ such that

$$
\mathcal{R}_{\mathbf{x}} f\left(x_{i}\right)=f\left(x_{i}\right), \quad i=1, \ldots, n .
$$

Thus, the definition of the remainder can be extended to any function $f(y)$, in particular, can be extended [15] to polynomials in $y, y^{-1}$, by requiring relations (7).

Similarly, the Bezoutian $\mathcal{B e z}_{\mathbf{x}}(f)$ of a function $f(y, z)$ is the matrix $\mathcal{B} e z_{\mathbf{x}}(f)=$ $\left[b_{i j}\right]_{i, j=0, \ldots, n-1}$, where $\sum_{i, j=0}^{n} b_{i j} z^{n-1-i} y^{n-j-1}$ is the remainder of $f(y, z)$ modulo $S_{n}(z-\mathbf{x})$ and modulo $S_{n}(y-\mathbf{x})[10,12$, Th.3.4.1].

Given another alphabet $\mathbf{c}=\left\{c_{1}, \ldots, c_{m}\right\}$, the resultant $R(\mathbf{x}, \mathbf{c})$ is defined to be

$$
R(\mathbf{x}, \mathbf{c})=\prod_{i=1}^{n} \prod_{j=1}^{m}\left(x_{i}-c_{j}\right)
$$

It is also [10, Th.3.2.1] equal to the Schur function $S_{m^{n}}(\mathbf{x}-\mathbf{c})$. 
Lemma 1. The determinant of $\mathcal{B} e z_{\mathbf{x}}\left(S_{n-1}(y+z) S_{m}(z-\mathbf{c})\right)$ is equal to the resultant $R(\mathbf{x}, \mathbf{c})$.

Proof. Instead of expanding the double remainder in the basis $y^{j} z^{i}$, let us choose the basis $y^{j} S_{i}(z-\mathbf{c}), i, j=0, \ldots, n-1$. One has

$$
\begin{aligned}
S_{n-1}(y+z) S_{m}(z-\mathbf{c}) & =\sum_{j=0}^{n-1} y^{n-1-j} z^{j} S_{m}(z-\mathbf{c})=\sum_{j=0}^{n-1} y^{n-1-j} S_{m+j}(z-\mathbf{c}) \\
& =\sum_{j=0}^{n-1} y^{n-1-j} S_{m+j}((z-\mathbf{x})+(\mathbf{x}-\mathbf{c})) \\
& \equiv \sum_{j=0}^{n-1} \sum_{i=0}^{n-1} y^{n-1-j} S_{i}(z-\mathbf{x}) S_{m+j-i}(\mathbf{x}-\mathbf{c})
\end{aligned}
$$

Hence, the Bezoutian, expressed in these bases, is the matrix $\left[S_{m+j-i}(\mathbf{x}-\mathbf{c})\right]$, the determinant of which is equal to $S_{m^{n}}(\mathbf{x}-\mathbf{c})$.

Using $\left\{c_{1}, \ldots, c_{m}, 0\right\}$ instead of $\mathbf{c}$, one obtains that the determinant of

$$
\mathcal{B} e z_{\mathbf{x}}\left(S_{n-1}(y+z) z S_{m}(z-\mathbf{c})\right)
$$

is equal to $x_{1} \cdots x_{n} R(\mathbf{x}, \mathbf{c})=S_{(m+1)^{n}}(\mathbf{x}-\mathbf{c})$, and more generally, that

$$
\operatorname{det}\left(\mathcal{B} e z_{\mathbf{x}}\left(S_{n-1}(y+z) z^{k} S_{m}(z-\mathbf{c})\right)\right)=S_{(m+k)^{n}}(\mathbf{x}-\mathbf{c})
$$

the equality being valid for $k \in \mathbb{Z}$, once its is checked for a single value of $k$.

For example, for $n=2=m$, the matrices of the remainders in the basis $\{1, y\} \otimes\{1, z-\mathbf{x}\}$ are, for $k=0,-1,-2$ respectively,

$$
\left[\begin{array}{ll}
S_{1}(\mathbf{x}-\mathbf{c}) & S_{2}(\mathbf{x}-\mathbf{c}) \\
S_{2}(\mathbf{x}-\mathbf{c}) & S_{3}(\mathbf{x}-\mathbf{c})
\end{array}\right],\left[\begin{array}{cc}
1-\frac{c_{1} c_{2}}{x_{1} x_{2}} & S_{1}(\mathbf{x}-\mathbf{c}) \\
S_{1}(\mathbf{x}-\mathbf{c}) & S_{2}(\mathbf{x}-\mathbf{c})
\end{array}\right],\left[\begin{array}{cc}
\frac{y_{1}+y_{2}}{x_{1} x_{2}}-\frac{y_{1} y_{2}\left(x_{1}+x_{2}\right)}{\left(x_{1} x_{2}\right)^{2}} & 1-\frac{c_{1} c_{2}}{x_{1} x_{2}} \\
1-\frac{c_{1} c_{2}}{x_{1} x_{2}} & S_{1}(\mathbf{x}-\mathbf{c})
\end{array}\right] .
$$

\subsection{Discriminants}

Let $f(y)=R(y, \mathbf{x})$. Write the derivative $f^{\prime}(y)$ of $f(y)$ in the factorized form $f^{\prime}(y)=n R\left(y, \mathbf{x}^{\text {der }}\right)$, using the alphabet $\mathbf{x}^{\text {der }}$ of roots of $f^{\prime}(y)$. The logarithmic derivative of $f^{\prime}(y)$ shows that $p_{k}(\mathbf{x})=n h_{k}\left(\mathbf{x}-\mathbf{x}^{\text {der }}\right)$ for any $k \geq 0$. Since for any $i,\left\{x_{i}^{r}\right\}$ is a recursive sequence satisfying $(4),\left\{p_{r}(\mathbf{x})=x_{1}^{r}+\cdots+x_{n}^{r}, r \in \mathbb{Z}\right\}$ is a recursive sequence satisfying (4). Thus the equality $p_{k}(\mathbf{x})=n h_{k}\left(\mathbf{x}-\mathbf{x}^{\text {der }}\right)$ can be extended to any $k \in \mathbb{Z}$ by taking the preceding conventions for $h_{k}(\mathbf{x})$ and $p_{k}(\mathbf{x}), k \in \mathbb{Z}$.

The resultant of $R\left(\mathbf{x}, \mathbf{x}^{\text {der }}\right)$ is equal to $S_{(n-1)^{n}}\left(\mathbf{x}-\mathbf{x}^{\text {der }}\right)$, that is, to the determinant $\left|\frac{1}{n} p_{n-1+j-i}(\mathbf{x})\right|_{i, j=1, \ldots, n}$, which is equal to $(-1)^{\left(\begin{array}{c}n \\ 2\end{array}\right)} \mathcal{D}(\mathbf{x}, 1)$, where $\mathcal{D}(\mathbf{x}, 1)$ is the square of the Vandermonde in $\mathbf{x}$, called the discriminant. 
More generally, the resultant $R(\mathbf{x}, q \mathbf{x})=\prod_{i, j=1, \ldots, n}\left(x_{i}-q x_{j}\right)=S_{n^{n}}(\mathbf{x}-q \mathbf{x})$

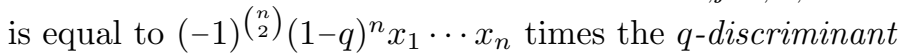

$$
\mathcal{D}(x, q)=\prod_{1 \leq i<j \leq n}\left(x_{i}-q x_{j}\right)\left(x_{j}-q x_{i}\right) .
$$

\section{Determinantal expressions}

It is clear that $\mathfrak{P f}(a, h, n, 0)$ is of degree $n$ in the variables $h_{0}, h_{1}, \ldots$, and thus expands as a sum of Schur functions of index in $\mathbb{N}^{n}$. One can therefore introduce $\mathbf{x}=\left\{x_{1}, \ldots, x_{n}\right\}$ and specialize each $h_{i}$ to the complete function $h_{i}(\mathbf{x})$ without loss of information (thanks to homogeneity, $h_{0}(\mathbf{x})=1$ creates no problem). Moreover, we have seen in the preceding section that shifting the indices $h_{i}(\mathbf{x}) \rightarrow h_{i+k}(\mathbf{x})$ with a fixed $k \in \mathbb{Z}$ multiplies the skew Schur functions of $\mathbf{x}$ by a factor $\left(x_{1} \cdots x_{n}\right)^{k}$. Hence, it is easy to pass from the Pfaffian in $\left(a_{i}-a_{j}\right) h_{i+j-3}(\mathbf{x})$ to the Pfaffian in $\left(a_{i}-a_{j}\right) h_{i+j-3+k}(\mathbf{x})$.

Let $E(\mathbf{x})$ be the matrix of order $2 n$ with entries the signed elementary symmetric functions $(-1)^{j-i} e_{j-i}(\mathbf{x})$ (defined to be 0 for negative indices). It is straightforward that sums of the type $\sum_{i=0}^{j}(-1)^{i} e_{j-i}(\mathbf{x}) h_{k+i}(\mathbf{x})$, for $k \geq n-1$, and $j \geq 0$, are equal to hook Schur functions $S_{1^{j}, k}(\mathbf{x})$.

Using this property, one checks the following proposition by decomposing linearly the Pfaffian matrix according to the $a_{i}$ 's.

Proposition 2. The matrix $E^{\operatorname{tr}}(\mathbf{x}) \mathfrak{M}(\mathbf{a}, h(\mathbf{x}), n, 0) E(\mathbf{x})$ is such that its submatrix on rows and columns $n+1, \ldots, 2 n$ is null. The submatrix on rows $1, \ldots, n$ and columns $n+1, \ldots, 2 n$, denoted by $\mathfrak{M}^{e}(\mathbf{a}, \mathbf{x}, n)$, is equal to

$$
\mathfrak{M}^{e}(\mathbf{a}, \mathbf{x}, n)=\sum_{r=1}^{2 n} a_{r}\left[(-1)^{n+r+i+j} e_{n-r+j}(\mathbf{x}) S_{1^{i-1}, r-2}(\mathbf{x})\right] \text {. }
$$

For example, for $n=2$, one has

$$
\begin{aligned}
& {\left[\begin{array}{cccc}
1 & 0 & 0 & 0 \\
-e_{1} & 1 & 0 & 0 \\
e_{2} & -e_{1} & 1 & 0 \\
0 & e_{2} & -e_{1} & 1
\end{array}\right]\left[\begin{array}{cccc}
0 & \left(a_{1}-a_{2}\right) S_{0} & \left(a_{1}-a_{3}\right) S_{1} & \left(a_{1}-a_{4}\right) S_{2} \\
\left(a_{2}-a_{1}\right) S_{0} & 0 & \left(a_{2}-a_{3}\right) S_{2} & \left(a_{2}-a_{4}\right) S_{3} \\
\left(a_{3}-a_{1}\right) S_{1} & \left(a_{3}-a_{2}\right) S_{2} & 0 & \left(a_{3}-a_{4}\right) S_{4} \\
\left(a_{4}-a_{1}\right) S_{2} & \left(a_{4}-a_{2}\right) S_{3} & \left(a_{4}-a_{3}\right) S_{4} & 0
\end{array}\right]\left[\begin{array}{cccc}
1 & -e_{1} & e_{2} & 0 \\
0 & 1 & -e_{1} & e_{2} \\
0 & 0 & 1 & -e_{1} \\
0 & 0 & 0 & 1
\end{array}\right]} \\
& =\left[\begin{array}{cccc}
0 & \left(a_{1}-a_{2}\right) S_{0} & \left(a_{2}-a_{3}\right) S_{1} & \left(a_{3}-a_{4}\right) S_{2}+\left(a_{3}-a_{2}\right) S_{11} \\
\left(a_{2}-a_{1}\right) S_{0} & 0 & \left(a_{3}-a_{1}\right) S_{11} & \left(a_{4}-a_{3}\right) S_{12} \\
\left(a_{3}-a_{2}\right) S_{1} & \left(a_{1}-a_{3}\right) S_{11} & 0 & 0 \\
\left(a_{4}-a_{3}\right) S_{2}+\left(a_{2}-a_{3}\right) S_{11} & \left(a_{3}-a_{4}\right) S_{12} & 0 & 0
\end{array}\right] .
\end{aligned}
$$

The $2 \times 2$ North-East corner $\mathfrak{M}^{e}(\mathbf{a}, \mathbf{x}, 2)$ expands as

$$
\begin{aligned}
& a_{1}\left[\begin{array}{ll}
-e_{2} S_{0,-1} & 0 \\
-e_{2} S_{1,-1} & 0
\end{array}\right]+a_{2}\left[\begin{array}{cc}
e_{1} S_{0,0} & -e_{2} S_{0,0} \\
e_{1} S_{1,0} & -e_{2} S_{1,0}
\end{array}\right]+a_{3}\left[\begin{array}{cc}
-e_{0} S_{0,1} & e_{1} S_{0,1} \\
e_{0} S_{1,1} & -e_{1} S_{1,1}
\end{array}\right]+a_{4}\left[\begin{array}{cc}
0 & -e_{0} S_{0,2} \\
0 & e_{0} S_{1,2}
\end{array}\right] \\
= & a_{1}\left[\begin{array}{cc}
0 & 0 \\
-e_{2} & 0
\end{array}\right]+a_{2}\left[\begin{array}{cc}
e_{1} & -e_{2} \\
0 & 0
\end{array}\right]+a_{3}\left[\begin{array}{cc}
-e_{1} & e_{1} S_{1} \\
e_{2} & -e_{2} S_{1}
\end{array}\right]+a_{4}\left[\begin{array}{cc}
0 & -S_{2} \\
0 & e_{1} S_{2}
\end{array}\right] .
\end{aligned}
$$

One can also write the Pfaffian as the determinant of a $2 n \times 2 n$ matrix in different manners, as shows the next result. 
Proposition 3. For a given $n$, let $\mathfrak{M}_{h}$ be the matrix of order $2 n$ with $i$-th row

$$
\left[h_{n+1-i}(\mathbf{x}), \ldots, h_{2 n-i}(\mathbf{x}), a_{n-i+1} h_{n+1-i}(\mathbf{x}), \ldots, a_{n-i+1} h_{2 n-i}(\mathbf{x})\right],
$$

let $\mathfrak{M}_{e}$ be the matrix obtained by changing each $h_{i}(\mathbf{x})$ to $e_{i}(\mathbf{x})$ in $\mathfrak{M}_{h}$, and finally, let $\mathfrak{M}_{x}$ be the matrix with $i$-th row

$$
\left[x_{1}^{2 n-i}, \ldots, x_{n}^{2 n-i}, a_{2 n-i+1} x_{1}^{2 n-i}, \ldots, a_{2 n-i+1} x_{n}^{2 n-i}\right] .
$$

Then $\mathfrak{P f}(\mathbf{a}, h(\mathbf{x}), n, 2-n)$ is equal to the determinant of $\mathfrak{M}_{h}$, of $\mathfrak{M}_{e}$, and equal to the quotient of the determinant of $\mathfrak{M}_{x}$ by the square of the Vandermonde determinant in $x_{1}, \ldots, x_{n}$.

Proof. It results from the analysis in [11] that the Pfaffian is determined by specializing a to a permutation of $1^{n} 0^{n}$ (in fact, Yamanouchi words suffice). In that case, the Pfaffian becomes equal, up to a sign, to some determinant of order of $n$ (denoted $g(i \cdots j \mid k \cdots \ell)$ in [11]). In the present case, this determinant is of the type $\left|h_{u_{i}+v_{j}+i+j-n-1}(\mathbf{x})\right|$, with $u, v$ two increasing partitions in $\mathbb{N}^{n}$. But such a determinant is equal to $(-1)^{\left(\begin{array}{l}n \\ 2\end{array}\right)} S_{u}(\mathbf{x}) S_{v}(\mathbf{x})$ thanks to (1). Similarly, the specializations of $\mathfrak{M}_{h}, \mathfrak{M}_{e}$ or $\mathfrak{M}_{x}$ give rise to products of two Schur functions.

For example, for $n=2$, the three matrices appearing in the proposition are

$$
\left[\begin{array}{cccc}
h_{2} & h_{3} & a_{4} h_{2} & a_{4} h_{3} \\
h_{1} & h_{2} & a_{3} h_{1} & a_{3} h_{2} \\
1 & h_{1} & a_{2} & a_{2} h_{1} \\
0 & 1 & 0 & a_{1}
\end{array}\right],\left[\begin{array}{cccc}
e_{2} & 0 & a_{4} e_{2} & 0 \\
e_{1} & e_{2} & a_{3} e_{1} & a_{3} e_{2} \\
1 & e_{1} & a_{2} & a_{2} e_{1} \\
0 & 1 & 0 & a_{1}
\end{array}\right],\left[\begin{array}{cccc}
x_{1}^{3} & x_{2}^{3} & a_{4} x_{1}^{3} & a_{4} x_{2}^{3} \\
x_{1}^{2} & x_{2}^{2} & a_{3} x_{1}^{2} & a_{3} x_{2}^{2} \\
x_{1} & x_{2} & a_{2} x_{1} & a_{4} x_{2} \\
1 & 1 & a_{1} & a_{1}
\end{array}\right],
$$

and the determinant of the first two matrices is equal to

$$
\mathfrak{P f}(\mathbf{a}, h(\mathbf{x}), 2,0)=\left(a_{3}-a_{2}\right)\left(a_{4}-a_{1}\right) S_{22}(\mathbf{x})-\left(a_{2}-a_{1}\right)\left(a_{4}-a_{3}\right) S_{13}(\mathbf{x}) .
$$

One can extend the preceding property by shifting indices: $h_{i} \rightarrow h_{i+r}$, and considering functions of $\mathbf{x}-\mathbf{y}$ instead of $\mathbf{x}$. Thus given $\mathbf{y}=\left\{y_{1}, \ldots, y_{m}\right\}$ of cardinality $m$, and any $r \in \mathbb{Z}$ let $\mathfrak{M}_{h}(\mathbf{a}, \mathbf{x}-\mathbf{y}, r)$ be the matrix of order $2 n$ with $i$-th row

$$
\begin{aligned}
{\left[h_{n+1-i+r}(\mathbf{x}-\mathbf{y}), \ldots,\right.} & h_{2 n-i+r}(\mathbf{x}-\mathbf{y}), \\
& \left.a_{n-i+1} h_{n+1-i+r}(\mathbf{x}-\mathbf{y}), \ldots, a_{n-i+1} h_{2 n-i+r}(\mathbf{x}-\mathbf{y})\right] .
\end{aligned}
$$

Thanks to (5), each minor on the first $n$ columns, or last $n$ columns of $\mathfrak{M}_{h}(\mathbf{a}, \mathbf{x}-\mathbf{y}, r)$ is equal to the product of the same minor of $\mathfrak{M}_{h}(\mathbf{a}, \mathbf{x}-\mathbf{y}, 0)$ by $R(\mathbf{x}, \mathbf{y})\left(x_{1} \cdots x_{n}\right)^{r-m}$. Similarly,

$$
\mathfrak{P} \mathfrak{f}(\mathbf{a}, h(\mathbf{x}-\mathbf{y}), n, k)=\mathfrak{P} \mathfrak{f}(\mathbf{a}, h(\mathbf{x}), n, 0) R(\mathbf{x}, \mathbf{y})\left(x_{1} \cdots x_{n}\right)^{k-m},
$$

since the Pfaffian is a linear combination of Schur functions. Hence the preceding proposition entails: 
Theorem 4. Given two finite alphabets $\mathbf{x}$ of cardinality $n, \mathbf{y}$ of cardinality $m$, and two integers $k, r \in \mathbb{Z}$ such that $k+m+n-2 r-2=0$, then one has

$$
\begin{aligned}
\mathfrak{P f}(\mathbf{a}, h(\mathbf{x}-\mathbf{y}), n, k) & =\left(x_{1} \cdots x_{n}\right)^{k-m} R(\mathbf{x}, \mathbf{y}) \mathfrak{P f}(\mathbf{a}, h(\mathbf{x}), n, 0) \\
& =\frac{1}{R(\mathbf{x}, \mathbf{y})} \operatorname{det}\left(\mathfrak{M}_{h}(\mathbf{a}, \mathbf{x}-\mathbf{y}, r)\right) .
\end{aligned}
$$

For example, for $n=2, m=1$, the matrix

$$
\mathfrak{M}_{h}(\mathbf{a}, \mathbf{x}-\mathbf{y}, 0)=\left[\begin{array}{cccc}
h_{2}(\mathbf{x}-\mathbf{y}) & h_{3}(\mathbf{x}-\mathbf{y}) & a_{4} h_{2}(\mathbf{x}-\mathbf{y}) & a_{4} h_{3}(\mathbf{x}-\mathbf{y}) \\
h_{1}(\mathbf{x}-\mathbf{y}) & h_{2}(\mathbf{x}-\mathbf{y}) & a_{3} h_{1}(\mathbf{x}-\mathbf{y}) & a_{3} h_{2}(\mathbf{x}-\mathbf{y}) \\
1 & h_{1}(\mathbf{x}-\mathbf{y}) & a_{2} & a_{2} h_{1}(\mathbf{x}-\mathbf{y}) \\
y_{1}\left(x_{1} x_{2}\right)^{-1} & 1 & a_{1} y_{1}\left(x_{1} x_{2}\right)^{-1} & a_{1}
\end{array}\right]
$$

has determinant equal to

$$
\left(x_{1}-y_{1}\right)^{2}\left(x_{2}-y_{1}\right)^{2}\left(\left(a_{1}-a_{3}\right)\left(a_{2}-a_{4}\right)-\left(a_{1}-a_{2}\right)\left(a_{3}-a_{4}\right) \frac{\left(x_{1}+x_{2}\right)^{2}}{x_{1} x_{2}}\right) \text {. }
$$

There is another way to evaluate a Hankel Pfaffian, when a is specialized to $\mathbf{q}=\left\{1, q, q^{2}, \ldots, q^{2 n-1}\right\}$. The next theorem shows that in that case the matrix $\mathfrak{M}^{e}(\mathbf{a}, \mathbf{x}, n)$ coincides with a Bezoutian.

Theorem 5. Given $\mathbf{x}$ of cardinality $n$, one has for $k \in \mathbb{Z}$

(12) $\mathfrak{M}^{e}\left(\left(q^{i-1}-q^{j-1}\right) h_{i+j+k-n-2}(\mathbf{x})\right)=\mathcal{B} e z_{\mathbf{x}}\left(-z^{k} S_{n-1}(q z+y) S_{n}(q z-\mathbf{x})\right)$.

Proof. Suppose $k \geq 0$ and expand

$$
\begin{aligned}
-z^{k} S_{n-1}(q z+y) S_{n}(q z-\mathbf{x}) & =-\sum_{j=0}^{n-1} y^{j} z^{k+n-1-j} q^{n-1-j} S_{n}(q z-\mathbf{x}) \\
& =-\sum_{j=0}^{n-1} q^{2 n-1-j} y^{j} S_{2 n+k-j-1}\left(z-q^{-1} \mathbf{x}\right) .
\end{aligned}
$$

Using the expression of the remainder as a hook Schur function given in [10, Th.3.2.1], one has

$$
S_{m}(z-B) \equiv \sum_{j=0}^{m} \sum_{i=0}^{n-1}(-z)^{i} S_{1^{n-1-i}, m-n+1-j}(\mathbf{x}) S_{j}\left(-q^{-1} \mathbf{x}\right) \quad \bmod R(z, \mathbf{x})
$$

and one obtains that the Bezoutian is a matrix with entries equal to hook Schur functions of $\mathbf{x}$ times functions $S_{j}\left(-q^{-1} \mathbf{x}\right)=(-1)^{j} q^{-j} e_{j}(\mathbf{x})$. More precisely, filtering the Bezoutian according to powers of $q$, one recognizes in this filtration exactly the filtration of $\mathfrak{M}^{e}(\mathbf{a}, n, k)$ according to $a_{1}, \ldots, a_{2 n}$ obtained from (9). The expression remains valid for $k<0$ because the entries of the Bezoutian for variable $k$ form a recursive sequence with the same characteristic polynomial as the sequence $h_{k}(\mathbf{x}), k \in \mathbb{Z}$. 
For example, for $n=2$ and $k=0$, one has

$$
\begin{aligned}
& \mathcal{B}_{\mathbf{x}}\left(-(q z+y) S_{2}(q z-\mathbf{x})\right) \\
= & \mathcal{B} e z_{\mathbf{x}}\left(-S_{3}(q z-\mathbf{x})-y S_{2}(q z-\mathbf{x})\right) \\
= & {\left[\begin{array}{cc}
0 & 0 \\
-e_{2} & 0
\end{array}\right]+q\left[\begin{array}{cc}
e_{1} & -e_{2} \\
0 & 0
\end{array}\right]+q^{2}\left[\begin{array}{cc}
-e_{1} & e_{1} S_{1} \\
e_{2} & -e_{2} S_{1}
\end{array}\right]+q^{3}\left[\begin{array}{cc}
0 & -S_{2} \\
0 & e_{1} S_{2}
\end{array}\right] . }
\end{aligned}
$$

Since $-q S_{n-1}(q z+y) S_{n}(q z-\mathbf{x})=-S_{n-1}\left(z+q^{-1} y\right) S_{n}\left(z-q^{-1} \mathbf{x}\right)$, the determinant of $\mathcal{B e z}_{\mathbf{x}}\left(S_{n-1}(q z+y) S_{n}(q z-\mathbf{x})\right)$ is equal to the resultant $R\left(\mathbf{x}, q^{-1} \mathbf{x}\right)$ up to a power of $q$ and a sign.

Controlling the power of $q$, and using the invariance of

$$
\operatorname{det}\left(\mathcal{B} e z_{\mathbf{x}}\left(-z^{k} S_{n-1}(q z+y) S_{n}(q z-\mathbf{x})\right)\right)\left(x_{1} \cdots x_{n}\right)^{-k}
$$

with respect to $k$, one obtains the following property.

Theorem 6. Given $\mathbf{x}$ of cardinality $n$, one has for $k \in \mathbb{Z}$

$$
\begin{aligned}
\mathfrak{P f}\left(\left(q^{i-1}-q^{j-1}\right) h_{i+j+k-n-1}(\mathbf{x})\right) & =(-1)^{\left(\begin{array}{c}
n \\
2
\end{array}\right)} S_{n^{n}}((1-q) \mathbf{x})\left(x_{1} \cdots x_{n}\right)^{k} \\
& =(-1)^{\left(\begin{array}{c}
n \\
2
\end{array}\right)}(1-q)^{n}\left(x_{1} \cdots x_{n}\right)^{k+1} \mathcal{D}_{\mathbf{x}}(q) .
\end{aligned}
$$

For example, for $n=3$, and $k=-1$, one has $h_{-1}(\mathbf{x})=0=h_{-2}(\mathbf{x})$ and the determinant of the skew-symmetric matrix

$$
\left[\begin{array}{cccccc}
0 & 0 & 0 & 1-q^{3} & \left(1-q^{4}\right) S_{1} & \left(1-q^{5}\right) S_{2} \\
0 & 0 & q-q^{2} & \left(q-q^{3}\right) S_{1} & \left(q-q^{4}\right) S_{2} & \left(q-q^{5}\right) S_{3} \\
0 & q^{2}-q & 0 & \left(q^{2}-q^{3}\right) S_{2} & \left(q^{2}-q^{4}\right) S_{3} & \left(q^{2}-q^{5}\right) S_{4} \\
q^{3}-1 & \left(q^{3}-q\right) S_{1} & \left(q^{3}-q^{2}\right) S_{2} & 0 & \left(q^{3}-q^{4}\right) S_{4} & \left(q^{3}-q^{5}\right) S_{5} \\
\left(q^{4}-1\right) S_{1} & \left(q^{4}-q\right) S_{2} & \left(q^{4}-q^{2}\right) S_{3} & \left(q^{4}-q^{3}\right) S_{4} & 0 & \left(q^{4}-q^{5}\right) S_{6} \\
\left(q^{5}-1\right) S_{2} & \left(q^{5}-q\right) S_{3} & \left(q^{5}-q^{2}\right) S_{4} & \left(q^{5}-q^{3}\right) S_{5} & \left(q^{5}-q^{4}\right) S_{6} & 0
\end{array}\right]
$$

is equal to the square of $(1-q)^{3} q^{3} \mathcal{D}_{\mathbf{x}}(q)$.

\section{Representations of the symmetric group}

To understand the dependency in a of the Pfaffian $\mathfrak{P f}(\mathbf{a}, \mathbf{h}, n)$, we need to use the theory of representations. Irreducible representations of the symmetric group $\mathfrak{S}_{n}$ over $\mathbb{C}$ are in bijection with partitions of $n$. One usually indexes bases by standard Young tableaux of a given shape $\lambda$. The tableaux of shape $\lambda$ can be considered as the vertices of a graph, two tableaux being connected by an edge of label $s_{i}$ if the two tableaux differ by the transposition of $i, i+1$.

Interpreting tableaux of shape $\lambda$ as products of Vandermonde determinants, each column $u=\left[u_{1}, \ldots, u_{r}\right]$ giving rise to the Vandermonde

$$
\Delta^{x}(u)=\prod_{1 \leq i<j \leq r}\left(x_{u_{i}}-x_{u_{j}}\right),
$$


one obtains the Specht basis of the irreducible representation of index $\lambda$ of the symmetric group. More generally, we shall call Specht basis any image of this basis in another copy of the same representation.

In [11], we have used a Young basis rather than a Specht basis to expand a Pfaffian of the type $\mathfrak{P f}\left(\left(a_{i}-a_{j}\right) g_{i, j}\right), 1 \leq i<j \leq 2 n, g_{i, j}=g_{j, i}$, observing that three symmetric groups are involved: the symmetric group permuting the $a_{i}$, the symmetric group acting on $g_{i, j}$, and the diagonal group acting simultaneously on the indices of $a_{i}$ and $g_{i, j}$.

In the case of a Hankel Pfaffian, it will be more illuminating to use several Kazhdan-Lusztig bases, corresponding to different spaces of polynomials. The original constructions of Kazhdan and Lusztig stand at the level of the Hecke algebra. Unfortunately, general irreducible representations are still not fully explicit. However the case of interest for Pfaffians is the case corresponding to Graßmannians [9], that is, the case of rectangular partitions of the type $[n, n]$, or $\left[2^{n}\right]$ that one can find in the literature under many disguises.

We shall need only a pair of bases, but prefer to be more complete and describe the Kazhdan-Lusztig bases of some other realizations of the same representation.

\subsection{Combinatorial objects}

Bases of irreducible representations of the symmetric group are usually encoded by standard Young tableaux of a given shape. In our case, the shape will be $[n, n]$ or its transpose $\left[2^{n}\right]$.

From a $2 \times n$ Young tableau, one reads two partitions, by subtracting to the bottom row, as a vector, the vector $[1,2, \ldots, n-1]$, and by subtracting to $[n+1, \ldots, 2 n]$ the top row. These two partitions are contained in the staircase partition $[n-1, \ldots, 1,0]$. We shall label bases by the partition $\lambda$ (written decreasingly) corresponding to the bottom row of the tableau.

Thus the Young tableau

\begin{tabular}{|l|l|c|c|c|c|}
\hline 3 & 6 & 7 & 9 & 11 & 12 \\
\hline 1 & 2 & 4 & 5 & 8 & 10
\end{tabular}$\Rightarrow \begin{aligned} {[7, \ldots, 12]-[3,6,7,9,11,12] } & =[4,2,2,1,0,0] \\
{[1,2,4,5,8,10]-[1, \ldots, 6] } & =[0,0,1,1,3,4]\end{aligned}$

will be replaced by $\lambda=[4,3,1,1]$.

To $\lambda$ one also associates a skew partition $\lambda=\left(\left[(n-1)^{n}\right]+\lambda \omega\right) / \lambda^{\sim}$, where $\lambda \omega$ means the increasing reordering of $\lambda$. For the running example, it is

$$
\lambda=\left(\left[5^{6}\right]+[0,0,1,1,3,4]\right) /[0,0,1,2,2,4]=[5,5,6,6,8,9] /[0,0,1,2,2,4] .
$$

Reading the border of the diagram of $\lambda$, one obtains a Yamanouchi word that on can represent in the plane as a Dyck path, 1 standing for a North-East 
step, 0 a South-East step. For $n=4, \lambda=[3,1]$ (figured in grey) one has

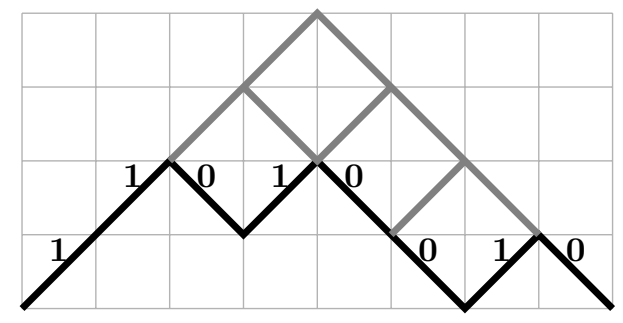

$$
\lambda=[3,1]
$$

Yamanouchi $[\mathbf{1}, \mathbf{1}, \mathbf{0}, \mathbf{1}, \mathbf{0}, \mathbf{0}, \mathbf{1}, \mathbf{0}]$

Pairing successively in the Yamanouchi word $1 \cdots 0$ treated as opening and closing parentheses, one obtains a link pattern. To a link between positions $i$ and $j$ one associates a factor $\left(a_{i}-a_{j}\right)$. Let $\varphi^{a}(\lambda)$ be the product of all such factors for the link pattern associated to $\lambda$. Equivalently, one labels the steps of the path by $1,2, \ldots, 2 n$, each factor $\left(a_{i}-a_{j}\right)$ corresponding to paired steps.

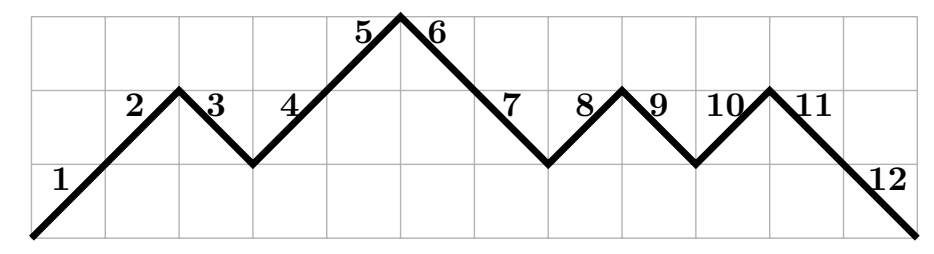

$$
\varphi^{a}([4,3,1,1])=\left(a_{1}-a_{12}\right)\left(a_{2}-a_{3}\right)\left(a_{4}-a_{7}\right)\left(a_{5}-a_{6}\right)\left(a_{8}-a_{9}\right)\left(a_{10}-a_{11}\right)
$$

Let $\psi(\lambda)$ be the vector obtained by labeling $0,2, \ldots, 2 n-2$ the successive increasing steps of the Dyck path, and labeling each descending step by the label of the step to which it is paired.

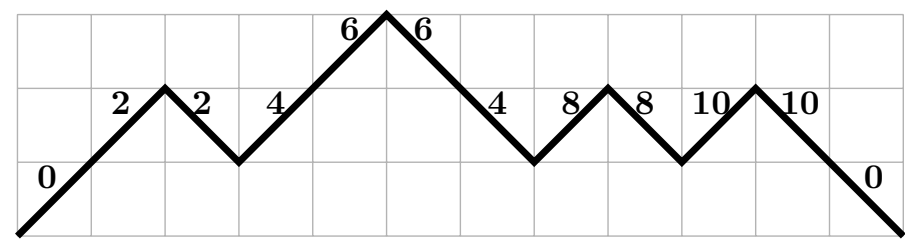

$$
\psi([4,3,1,1])=[0,2,2,4,6,6,4,8,8,10,10,0] .
$$

Given $n$ and a partition $\lambda$ one labels the boxes of the diagram of $\lambda$ by a pair of numbers. The first one increases by 1 when moving horizontally rightwards, and decreases by 1 when moving vertically downwards, starting from $n$ in the first box. The second number is 0 for the boxes in the corners, 1 for the new corners obtained by erasing the preceding corners, and so on. Let us denote 
this bi-labelled diagram $\mathcal{D}_{\lambda}$. A similar construction is given in type $B$ by [4].

$$
\mathcal{D}_{4311}=\begin{array}{|l|l|l|l|}
\hline 6,3 & 7,2 & 8,1 & 9,0 \\
\cline { 1 - 2 } 5,2 & 6,1 & 7,0 & \\
\cline { 1 - 2 } 4,1 & \multicolumn{3}{|l}{} \\
\cline { 1 - 1 } 3,0 & \multicolumn{2}{|l}{} \\
\cline { 1 - 1 } &
\end{array}
$$

The weights $\varphi^{a}(\lambda)$ and $\psi(\lambda)$ will be interpreted as dual Kazhdan-Lusztig bases, while the diagrams $\mathcal{D}_{\lambda}$ will be used to generate several Kazhdan-Lusztig bases [8].

\subsection{Basis $K L_{\lambda}^{\Delta}$}

Denoting $\Delta^{x}(u \mid v)$ the product of the two Vandermonde $\Delta^{x}(u), \Delta^{x}(v)$, one generates the basis $K L_{\lambda}^{\Delta}$ starting from $K L_{0}^{\Delta}:=\Delta^{x}(1 \cdots n \mid n+1 \cdots 2 n)$. The polynomial $K L_{\lambda}^{\Delta}$ is defined to be the image of $K L_{0}^{\Delta}$ under $\mathcal{D}_{\lambda}$, the diagram being read by successive rows, each entry $[i, k]$ being interpreted as $s_{i}-(1+k)^{-1}$, with $s_{i}$ the simple reflection exchanging $x_{i}, x_{i+1}$.

For example, for $n=3$, one has

$$
\begin{aligned}
K L_{11}^{\Delta} & =\Delta^{x}(123 \mid 456)\left(s_{3}-\frac{1}{2}\right)\left(s_{2}-1\right) \\
& =\Delta^{x}(123 \mid 456)\left(s_{3} s_{2}-s_{3}-\frac{1}{2}\left(s_{2}-1\right)\right) \\
& =\Delta^{x}(134 \mid 256)-\Delta^{x}(124 \mid 356)+\Delta^{x}(123 \mid 456) \\
& =\Delta^{x}(234 \mid 156)
\end{aligned}
$$

the last expression being due to the Plücker relations.

The full basis for $n=3$ is

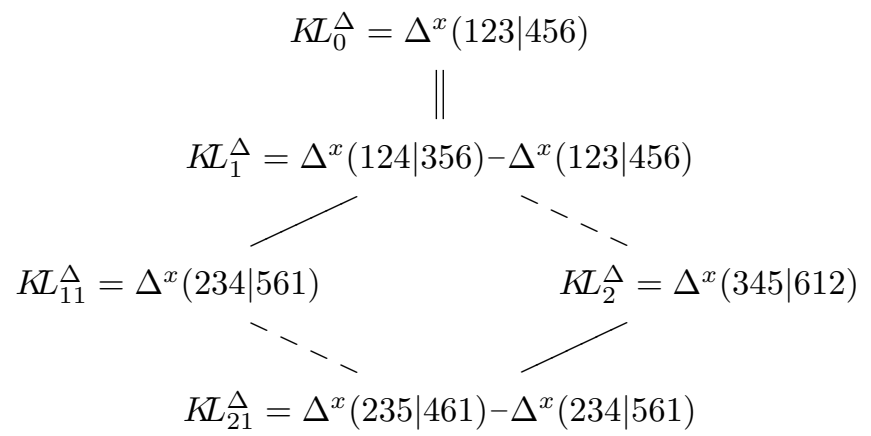




\subsection{Basis $K L_{\lambda}^{S}$}

The family of skew Schur functions $S_{\lambda}, \lambda \leq \rho$, is the Specht basis of an irreducible representation of index $\left[2^{n}\right]$. One interprets now standard tableaux as skew Schur functions, instead of products of Vandermonde determinants, keeping the same action of the symmetric group.

The Kazhdan-Lusztig basis $K L_{\lambda}^{S}$ is obtained from $K L_{0}^{S}=S_{(n-1)^{n}}$ using the diagrams $\mathcal{D}_{\lambda}, \lambda \leq \rho$, interpreting an entry $[i, k]$ as $s_{i}-(1+k)^{-1}$.

For example, for $n=3$, the Specht basis is $S_{0}=S_{222}, S_{1}=S_{223 / 001}$, $S_{\frac{11}{11}}=S_{233 / 002}, S_{2}=S_{224 / 011}, S_{21}=S_{234 / 012}$.

$$
\begin{aligned}
K L_{1}^{S} & =S_{222}\left(s_{3}-1\right)=S_{223 / 001}-S_{222}=S_{123} \\
K L_{2}^{S} & =S_{222}\left(s_{3}-\frac{1}{2}\right)\left(s_{4}-1\right)=S_{224 / 011}-S_{223 / 001}+S_{222}=S_{114} \\
K L_{11}^{S} & =S_{222}\left(s_{3}-\frac{1}{2}\right)\left(s_{2}-1\right)=S_{233 / 002}-S_{223 / 001}+S_{222}=S_{033} \\
K L_{21}^{S} & =S_{222}\left(s_{3}-\frac{1}{2}\right)\left(s_{2}-1\right)\left(s_{4}-1\right) \\
& =S_{234 / 012}-S_{224 / 011}-S_{233 / 002}+S_{223 / 001}-2 S_{222}=S_{024}+S_{123} .
\end{aligned}
$$

In short, the Specht basis and K-L basis for $n=3$ are
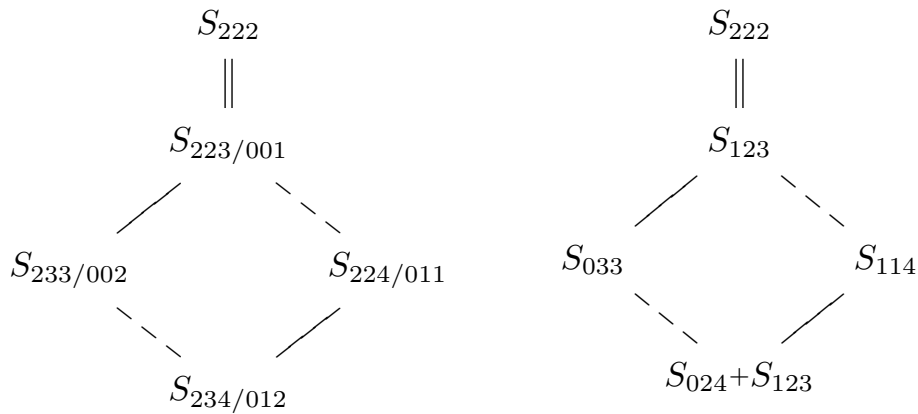

It seems a problem of interest for combinatorists to give the explicit expression of $K L_{\lambda}^{S}$ in terms of Schur functions. The following lemma describes the case where $K L_{\lambda}^{S}$ coincides with a single Schur function.

Lemma 7. Let $\lambda=\left[\beta^{\alpha}\right], \beta+\alpha \leq n$ be a rectangular partition. Then

$$
K L_{\lambda}^{S}=S_{(n-1-\alpha)^{\beta},(n-1)^{n-\alpha-\beta},(n-1+\beta)^{\alpha}} .
$$

Proof. In the case of a rectangular partition, the Kazhdan-Lusztig polynomials are trivial (i.e., equal to 1) [9]. In our terms, this translates into the fact that $K L_{\lambda}^{S}$ is the alternating sum of the elements of the Specht basis on the interval 
of partitions contained in $\lambda$ :

$$
K L_{\lambda}^{S}=\sum_{\mu \leq \lambda}(-1)^{|\mu|} S_{\left((n-1)^{n}+\mu\right) / \mu^{\sim}}
$$

To compute this sum, one may suppose that $h_{i}=h_{i}(\mathbf{x})$, with $\mathbf{x}$ of cardinality $n$. The skew Schur functions in the RHS factorize into $S_{(n-1)^{n} / \mu^{\sim}}(\mathbf{x}) S_{\mu}(\mathbf{x})$, according to (2).

To avoid elaborate manipulations of determinants, let us use the operator $\pi_{\omega}$ which sends $x^{v}: v \in \mathbb{N}^{n}$ onto $S_{v_{n}, \ldots, v_{1}}(\mathbf{x})$. One can now rewrite the RHS into

$$
\begin{aligned}
& \sum_{\nu \leq \alpha^{\beta}}(-1)^{|\nu|} \overbrace{x^{n-1, \ldots, n-1, n-1-\nu_{1}, \ldots, n-1-\nu_{\beta}}}^{n-\beta} S_{\nu \sim}(\mathbf{x}) \pi_{\omega} \\
= & \overbrace{}^{\overbrace{}^{n-1, \ldots, n-1, n-1-\alpha, \ldots, n-1-\alpha}} \overbrace{\beta^{\alpha}}\left(\mathbf{x}-x_{n-\beta+1}-\cdots-x_{n}\right) \pi_{\omega} \\
= & x^{(n-1)^{n-\beta},(n-1-\alpha)^{\beta}} x^{\beta^{\alpha}, 0^{n-\alpha}} \pi_{\omega}=S_{(n-1-\alpha)^{\beta},(n-1)^{n-\alpha-\beta},(n-1+\beta)^{\alpha}}(\mathbf{x}) .
\end{aligned}
$$

This is the required identity.

\subsection{Basis $K L_{\lambda}^{x}$}

One generates it from $K L_{0}^{x}=x_{1} \cdots x_{n}$, using the diagrams $\mathcal{D}_{\lambda}$, interpreting an entry $[i, k]$ as $s_{i}+(1+k)^{-1}$.

$$
\begin{gathered}
K L_{0}^{x}=x^{111000} \\
K L_{11}^{x}=x_{1}^{x}=x^{110100}+x^{111000} \\
K L_{21}^{x}=x^{10101}+x^{11001} \\
+x^{1011}+x^{1101}+2 x^{111}
\end{gathered}
$$

The coefficients are specializations $t=1$ of some Kazhdan-Lusztig polynomials, which are, in the case of Graßmannians, easy to compute [9]. In the preceding example, there is only one non trivial Kazhdan-Lusztig polynomial, and it is equal to $1+t$. This explains the coefficient 2 in the expansion of $K L_{21}^{x}$.

\subsection{Dual basis $L K_{\lambda}^{a}$}

One generates it using the reversed graph, with edges $s_{i}-1$, starting from

$$
L K_{\rho}^{a}=\Delta^{a}(12|34| \cdots \mid 2 n-1,2 n):=\left(a_{1}-a_{2}\right)\left(a_{3}-a_{4}\right) \cdots\left(a_{2 n-1}-a_{2 n}\right) .
$$


Notice that $L K_{\rho}^{a}$ is equal to the weight $\varphi^{a}(\rho)$. This equality transfers in fact to all partitions.

Lemma 8. For any $\lambda \leq \rho$, one has $\operatorname{LK}_{\lambda}^{a}=\varphi^{a}(\lambda)$.

Proof. The recursive definition of $L K_{\lambda}^{a}$ implies steps of the type

$$
\Delta^{a}(\cdots|j, i| i+1, k \mid \cdots) \stackrel{s_{i}-1}{\longrightarrow} \Delta^{a}(\cdots|j, i+1| i, k \mid \cdots)-\Delta^{a}(\cdots|j, i| i+1, k \mid \cdots) .
$$

But, thanks to the Plücker relations for minors of order 2, this last element is equal to $\Delta^{a}(\cdots|j, k| i, i+1 \mid \cdots)$. Therefore, the required property is true by decreasing induction on $\lambda$.

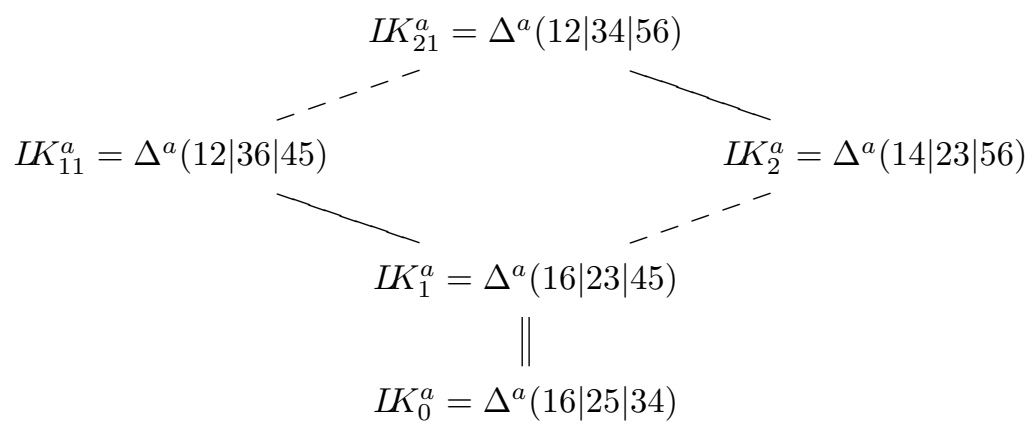

\subsection{Dual basis $L K_{\lambda}^{x}$}

One defines elements in the $\operatorname{ring} \mathcal{H}_{2 n}=\mathbb{Z}\left[x_{1}, \ldots, x_{2 n}\right] / \mathfrak{S y m}_{+}$, where $\mathfrak{S n m}_{+}$ is the ideal generated by symmetric polynomials without constant term. On this ring, one has a non-degenerate quadratic form $(,)^{\partial}$ such that $\left(x^{u}, x^{v}\right)^{\partial}=$ $(-1)^{\ell(\sigma)}$ if there exists a permutation $\sigma$ such that $(u+v)^{\sigma}=[2 n-1, \ldots, 1,0]$, and $\left(x^{u}, x^{v}\right)^{\partial}=0$ otherwise [10].

One generates in $\mathcal{H}_{2 n}$ a family $L K_{\lambda}^{x}, \lambda \leq \rho$, using the reversed graph, with edges $-\left(s_{i}+1\right)$, starting from $L K_{\rho}^{x}=x^{0022 \ldots, 2 n-2,2 n-2}$.

Thanks to the ideal, the dual basis can be represented by single monomials :

Lemma 9. For any $\lambda \leq \rho$, one has $L K_{\lambda}^{x}=x^{\psi(\lambda)}$.

Proof. An elementary step $L K_{\lambda}^{x} \rightarrow L K_{\mu}^{x}$ in the recursive definition corresponds to suppressing a corner labelled $[i, 0]$ in $\mathcal{D}_{\lambda}$, and for the corresponding weight $\psi(\lambda)$, to the transformation

$$
\begin{aligned}
& \psi(\lambda)=w a w^{\prime} a b w^{\prime \prime} b w^{\prime \prime \prime}, \\
& \psi(\mu)=w a w^{\prime} b b w^{\prime \prime} a w^{\prime \prime \prime},
\end{aligned}
$$

where the box stands in position $i, i+1$ and $a, b$ are two integers. We claim that

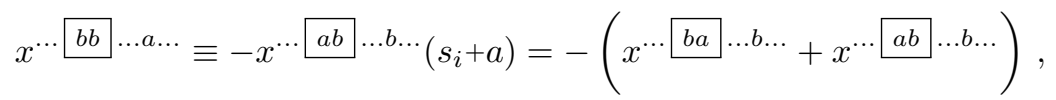


taking the notational liberty of replacing the common components of the two vectors by dots. By permutation, one can shift the varying components to the first three positions, and the property to show becomes

$$
x^{b b a \cdots}+x^{b a b \cdots}+x^{a b b \cdots} \equiv 0
$$

the three monomials differing only in the exponents of $x_{1}, x_{2}, x_{3}$. The nullity of the sum of three monomials can be tested by checking the scalar products with all monomials $x^{v}$. To hope for a permutation of $[2 n-1, \ldots, 0]$, the exponent $v$ must belong to $\{0,1\}^{2 n}$. But in that case the polynomial $\left(x^{b b a \cdots}+x^{b a b \cdots}+\right.$ $\left.x^{a b b \ldots}\right) x^{v}$ has at least a symmetry in $x_{1}, x_{2}$, or $x_{1}, x_{3}$, or $x_{2}, x_{3}$ and therefore $\left(x^{b b a \cdots}+x^{b a b \cdots}+x^{a b b \cdots}, x^{v}\right)^{\partial}$ is null even for those $v$.

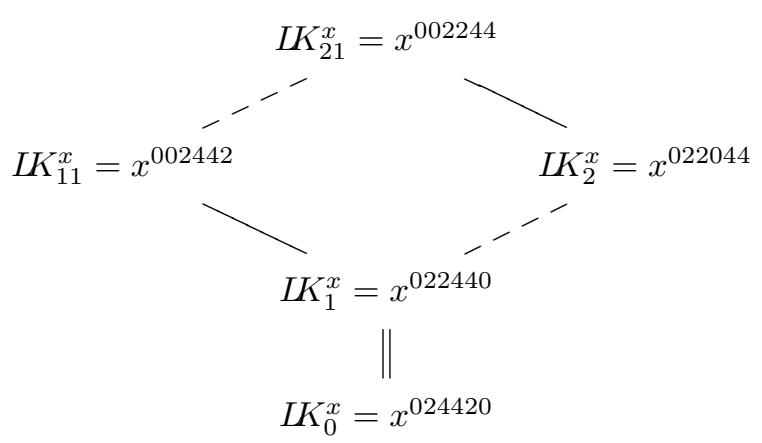

\subsection{Duality $K L_{\lambda}^{\Delta}, L K_{\lambda}^{x}$}

It remains to justify the terminology "dual basis", which does not reduce to reversing graphs. It is natural to use the vanishing properties of Vandermonde determinants, and, thus, to specialize the polynomials $K L_{\lambda}^{\Delta}$. In the present case, the following proposition shows that $\psi(0), \ldots, \psi(\rho)$ are convenient interpolation points.

Proposition 10. For any $\lambda, \mu \leq \rho$, one has

$$
K L_{\lambda}^{\Delta}(\psi(\mu))=(-1)^{|\lambda|-|\mu|} c_{n} \delta_{\lambda, \mu},
$$

with $c_{n}=\prod_{1 \leq i<j \leq n}(2 i-2 j)^{2}$.

Proof. Let us denote $\left(f, x^{v}\right)$ the evaluation of a function $f\left(x_{1}, \ldots, x_{2 n}\right)$ in $x_{1}=$ $v_{1}, \ldots, x_{2 n}=v_{2 n}$. This form is compatible with the action of the symmetric group: $\left(f s_{i}, g\right)=\left(f, g s_{i}\right)$.

On the other hand, given three exponents which differ only in three places, of the type $[\ldots b b \ldots a \ldots],[\ldots b a \ldots b \ldots],[\ldots a b \ldots b \ldots]$, one has

$$
\left(\Delta^{x}(1 \ldots n \mid n+1 \ldots 2 n), x^{\ldots b b \ldots a \ldots}+x^{\ldots b a \ldots b \ldots}+x^{\ldots a b \ldots b \ldots}\right)=0
$$

and therefore, for any $\lambda \leq \rho$,

$$
\left(K L_{\lambda}^{\Delta}, x^{\ldots b b \ldots a \cdots}+x^{\ldots b a \ldots b \cdots}+x^{\ldots a b \ldots b \cdots}\right)=0 .
$$


Starting with $\left(K L_{\lambda}^{\Delta}, x^{002244 \ldots}\right)=c_{n} \delta_{\lambda, \rho}$, one supposes that for some $\mu$ the proposition is true. Let $[i, 0]$ be a corner of $\mathcal{D}_{\mu}$, and let $\nu$ be the partition obtained from $\mu$ by removing this corner. Then

$$
\left(K L_{\lambda}^{x}, L K_{\nu}^{x}\right)=\left(K L_{\lambda}^{x},-L K_{\mu}^{x}\left(s_{i}+1\right)\right)=-\left(K L_{\lambda}^{x}\left(s_{i}+1\right), L K_{\mu}^{x}\right)
$$

thanks to (17) and Lemma 9. Non nullity can occur only for $\lambda=\mu$ or $\lambda=\nu$. Since $K L_{\nu}^{x}\left(s_{i}-1\right)=K L_{\mu}^{x}+\sum_{\eta \neq \mu, \nu} c_{\eta} K L_{\eta}^{x}$, one has

$$
\begin{aligned}
& -\left(K L_{\nu}^{x}\left(s_{i}+1\right), L K_{\mu}^{x}\right)=-\left(K L_{\mu}^{x}, L K_{\mu}^{x}\right), \\
& -\left(K L_{\mu}^{x}\left(s_{i}+1\right), L K_{\mu}^{x}\right)=-\left(K L_{\nu}^{x}\left(s_{i}-1\right)\left(s_{i}+1\right), L K_{\mu}^{x}\right)=0,
\end{aligned}
$$

which is what is expected for the proof by induction to be valid.

\subsection{Duality $K L_{\lambda}^{x}, L K_{\lambda}^{x}$}

All the products $K L_{\lambda}^{x} I K_{\mu}^{x}$ have total degree $0+1+\cdots+(2 n-1)$. This points to using the form $(,)^{\partial}[2]$.

Proposition 11. For any $\lambda, \mu \leq \rho$, one has

$$
\left(K L_{\lambda}^{x}, L K_{\mu}\right)^{\partial}=\delta_{\lambda, \mu}
$$

Proof. The starting point is $\left(x^{1 \ldots 10 \ldots 0}, L K_{\mu}\right)^{\partial}=\delta_{0, \mu}$. The general case is deduced by the same induction as in the preceding case, using, for any $f, g$, any $i \leq 2 n-1$ the identity $\left(f, g s_{i}\right)^{\partial}=-\left(f s_{i}, g\right)^{\partial}$.

\subsection{Duality $K L_{\lambda}^{x}, L K_{\lambda}^{a}$}

This time, we shall use the vanishing properties of the polynomials $L K_{\lambda}^{a}$.

Proposition 12. For any $\lambda, \mu \leq \rho$, one has

$$
\left(L K_{\lambda}^{a}, K L_{\mu}^{x}\right)=\delta_{\lambda, \mu}
$$

Proof. The starting point is $L K_{\mu}^{a}\left(x^{1 \ldots 10 \ldots 0}\right)=\delta_{0, \mu}$ and the proof by induction goes as before.

\section{Hankel Pfaffians in terms of Kazhdan-Lusztig basis}

In $[11$, Th.4.1], we have given several expressions of a Pfaffian with entries $\left(a_{i}-a_{j}\right) g_{i, j}$

For the present case, for $n=3$, this would read in the Specht basis as

$$
\mathfrak{P f}(\mathbf{a}, h(\mathbf{x}), 3,0)
$$

$$
\begin{aligned}
& =-\begin{array}{|l|l|l|}
\hline 4 & 5 & 6 \\
\hline 1 & 2 & 3
\end{array} S_{444}(\mathbf{x})+\begin{array}{|l|l|l|}
\hline 3 & 5 & 6 \\
\hline 1 & 2 & 4 \\
\hline
\end{array} S_{445 / 001}(\mathbf{x})-\begin{array}{|l|l|l|}
\hline 3 & 4 & 6 \\
\hline 1 & 2 & 5 \\
\hline
\end{array} S_{446 / 011}(\mathbf{x}) \\
& -\begin{array}{|l|l|l|}
\hline 2 & 5 & 6 \\
\hline 1 & 3 & 4 \\
\hline
\end{array} S_{455 / 002}(\mathbf{x})+\begin{array}{|l|l|l|}
\hline 2 & 4 & 6 \\
\hline 1 & 3 & 5 \\
\hline
\end{array}\left(S_{456 / 012}(\mathbf{x})-S_{444}(\mathbf{x})\right),
\end{aligned}
$$

each tableau being interpreted as a product of factors $\left(a_{i}-a_{j}\right)$ corresponding to its columns. 
In [11], we have shown in particular that the Pfaffian is diagonal in terms of Young's orthonormal basis. The underlying quadratic form in that case is formally defined in terms of tableaux [14, 2], but corresponds to the form $(,)^{\partial}$ when interpreted in the appropriate spaces. Therefore, thanks to (18), the Pfaffian remains diagonal when using Kazhdan-Lusztig bases instead of Young's bases [16], and one has the following theorem.

Theorem 13. Given n, then one has

$$
\mathfrak{P f}(\mathbf{a}, \mathbf{h}, n, 1-n)=\sum_{\lambda \leq \rho}(-1)^{|\lambda|} L K_{\lambda}^{a} K L_{\lambda}^{S} .
$$

For example, for $n=3$, one has

$$
\begin{aligned}
& \mathfrak{P} \mathfrak{f}(\mathbf{a}, \mathbf{h}, 3,-2) \\
= & \Delta^{a}(16|25| 34) K L_{0}^{S}-\Delta^{a}(16|23| 45) K L_{1}^{S}+\Delta^{a}(14|23| 56) K L_{2}^{S} \\
& +\Delta^{a}(12|36| 45) K L_{11}^{S}-\Delta^{a}(12|34| 56) K L_{21}^{S} \\
= & \left(a_{1}-a_{6}\right)\left(a_{2}-a_{5}\right)\left(a_{3}-a_{4}\right) S_{222}-\left(a_{1}-a_{6}\right)\left(a_{2}-a_{3}\right)\left(a_{4}-a_{5}\right) S_{123} \\
& +\left(a_{1}-a_{4}\right)\left(a_{2}-a_{3}\right)\left(a_{5}-a_{6}\right) S_{114}+\left(a_{1}-a_{2}\right)\left(a_{3}-a_{6}\right)\left(a_{4}-a_{5}\right) S_{033} \\
& -\left(a_{1}-a_{2}\right)\left(a_{3}-a_{4}\right)\left(a_{5}-a_{6}\right)\left(S_{024}+S_{123}\right) .
\end{aligned}
$$

\section{Discriminants and Bezoutians}

The case where a specializes to $\mathbf{q}=\left[1, q, \ldots, q^{2 n-1}\right]$ is of special interest. The Pfaffian $\mathfrak{P f}(\mathbf{q}, h(\mathbf{x}-\mathbf{y}), n, k)$ is proportional $\mathfrak{P} \mathfrak{f}(\mathbf{q}, h(\mathbf{x}), n, 2-n)$, which is equal to the determinant of the matrix $\mathfrak{M}_{h}(\mathbf{q}, \mathbf{x}, 0)$. But, because of homogeneity, one does not change the value of this determinant by replacing the entries $a_{i} h_{j}(\mathbf{x})=q^{i-1} h_{j}(\mathbf{x})$ by $q^{j} h_{j}(\mathbf{x})=h_{j}(q \mathbf{x})$. The Laplace expansion of this new matrix along the first $n$ columns is equal to $q^{\left(\begin{array}{c}n \\ 2\end{array}\right)}$ times the expansion of $S_{n^{n}}(\mathbf{x}-q \mathbf{x})=R(\mathbf{x}, q \mathbf{x})=(1-q)^{n}\left(x_{1} \cdots x_{n}\right) \mathcal{D}(\mathbf{x}, q)$.

In final, one has the following theorem linking Pfaffians, resultants and discriminants.

Theorem 14. Given $\mathbf{x}$ of cardinality $n, \mathbf{y}$ of cardinality $m$, and $k \in \mathbb{Z}$, then

$$
\mathfrak{P f}(\mathbf{q}, h(\mathbf{x}-\mathbf{y}), n, k)=q^{\left(\begin{array}{c}
n \\
2
\end{array}\right)}(1-q)^{n}\left(x_{1} \cdots x_{n}\right)^{k+n-1-m} R(\mathbf{x}, \mathbf{y}) \mathcal{D}(\mathbf{x}, q) .
$$

Consequently, one can use the expression of the Pfaffian in terms of the KL-basis to expand the $q$-discriminant.

For example, for $n=2,3,4$, denoting $[i]$ the $q$-integer $\left(q^{i}-1\right) /(q-1)$, one has the following expansions:

$$
\begin{aligned}
\mathcal{D}(2, q)= & {[3] K L_{0}^{S}-q K L_{1}^{S}=\left(1+q+q^{2}\right) S_{11}-q S_{02} } \\
\mathcal{D}(3, q)= & {[3][5] K L_{0}^{S}-q[5] K L_{1}^{S}+q[3]^{2} K L_{2}^{S}+q^{2}[3] K L_{11}^{S}-q^{3} K L_{12}^{S} } \\
= & \left(1+q+q^{2}\right)\left(1+\cdots+q^{4}\right) S_{222}-q\left(1+\cdots+q^{4}\right) S_{123}+q^{2}\left(1+q+q^{2}\right) S_{114} \\
& +q^{2}\left(1+q+q^{2}\right) S_{033}-q^{3}\left(S_{024}+S_{123}\right)
\end{aligned}
$$




$$
\begin{aligned}
\mathcal{D}(4, q)= & {[3][5][7] K L_{0}^{S}-q[5][7] K L_{1}^{S}+q^{2}[3][7] K L_{2}^{S}-q^{3}[3][5] K L_{3}^{S} } \\
& +q^{2}[3][7] K L_{11}^{S}-q^{3}[7] K L_{21}^{S}-q^{3}[3][5] K L_{111}^{S} \\
& +q^{4}[3]^{2} K L_{22}^{S}+q^{4}[5] K L_{31}^{S}+q^{4}[5] K L_{211}^{S} \\
& -q^{5}[3] K L_{32}^{S}-q^{5}[3] K L_{221}^{S}-q^{5}[3] K L_{311}^{S}+q^{6} K L_{321}^{S} .
\end{aligned}
$$

Since one has $p_{r}(\mathbf{x})=n h_{r}\left(\mathbf{x}-\mathbf{x}^{\text {der }}\right)$, one obtains from (21) the evaluation of Pfaffians where the power sums replace the complete functions, the resultant being replaced by the discriminant.

Corollary 15. Given $\mathbf{x}$ of cardinality $n$ and $k \in \mathbb{Z}$, then

$$
\mathfrak{P} \mathfrak{f}(\mathbf{q}, \mathbf{p}(\mathbf{x}), n, k)=(-q)^{\left(\begin{array}{l}
n \\
2
\end{array}\right)}(1-q)^{n}\left(x_{1} \cdots x_{n}\right)^{k} \mathcal{D}(\mathbf{x}, 1) \mathcal{D}(\mathbf{x}, q) .
$$

The limit $q \rightarrow 1$ gives that the Pfaffian with entries $(i-j) p_{i+j-3+k}(\mathbf{x})$ is equal to

$$
\left(x_{1} \cdots x_{n}\right)^{k+n-1-m} \mathcal{D}(\mathbf{x}, 1) \mathcal{D}(\mathbf{x}, q)
$$

and that the Pfaffian with entries $(i-j) p_{i+j-3+k}(\mathbf{x})$ is equal to

$$
(-1)^{\left(\begin{array}{c}
n \\
2
\end{array}\right)}\left(x_{1} \cdots x_{n}\right)^{k} \mathcal{D}(\mathbf{x}, 1)^{2} \text {. }
$$

One can also use the matrix $\mathfrak{M}_{h}\left(\mathbf{q}, \mathbf{x}-\mathbf{x}^{d e r}, k\right)$. For example, for $n=2$, $k=0$, one has

$$
\left|\begin{array}{cccc}
x_{1}^{2}+x_{2}^{2} & x_{1}^{3}+x_{2}^{3} & q^{3}\left(x_{1}^{2}+x_{2}^{2}\right) & q^{3}\left(x_{1}^{3}+x_{2}^{3}\right) \\
x_{1}+x_{2} & x_{1}^{2}+x_{2}^{2} & q^{2}\left(x_{1}+x_{2}\right) & q^{2}\left(x_{1}^{2}+x_{2}^{2}\right) \\
2 & x_{1}+x_{2} & 2 q & q\left(x_{1}+x_{2}\right) \\
\frac{1}{x_{1}}+\frac{1}{x_{2}} & 2 & \frac{1}{x_{1}}+\frac{1}{x_{2}} & 2
\end{array}\right|=\frac{q(q-1)^{2}}{x_{1} x_{2}}\left(x_{1}-x_{2}\right)^{4}\left(x_{1}-q x_{2}\right)\left(x_{2}-q x_{1}\right) .
$$

\section{Remark about Macdonald polynomials}

The KL-basis for the representations of shape $\left[2^{n}\right]$ or $[n, n]$ of the Hecke algebra is related to the non-symmetric Macdonald polynomials $M_{v}\left(\mathbf{x}_{2 n} ; t, q\right)$, $v \in \mathbb{N}^{2 n}$. In [3], one finds a common deformation of the KL basis and the Macdonald polynomials indexed by a permutation of [..221100], when $q$ is specialized to a certain root of $t$.

The $t$-discriminant itself, which is a symmetric function, is equal to the specialization of the symmetric Macdonald polynomial indexed by the (decreasing) partition $[2 n-2, \ldots, 2,0][1$, Th.3.2]. The $t$-discriminants also appear as specializations of symmetric Macdonald polynomial indexed by rectangular partitions [13, Remark 4.9].

Investigating extensively the specializations of symmetric or non-symmetric Macdonald polynomials at $q=t^{\alpha}$ seems to be of great interest.

\section{References}

[1] A. Boussicault and J.-G. Luque, Staircase Macdonald polynomials and the q-discriminant, DMTCS proceedings, (FPSAC 2008), arxiv:0801.2443 (2008). 
[2] C. Carré, A. Lascoux, and B. Leclerc, Turbo-straightening for decomposition into standard bases, Internat. J. Algebra Comput. 2 (1992), no. 3, 275-290.

[3] J. de Gier, A. Lascoux, and M. Sorrell, Deformed Kazhdan-Lusztig elements and Macdonald polynomials, J. Combin. Theory Ser. A 119 (2012), 183-211.

[4] J. de Gier and P. Pyatov, Factorised solutions of Temperley-Lieb qKZ equations on a segment, arxiv:0710.5362 (2007).

[5] M. Ishikawa, H. Tagawa, and J. Zeng, Pfaffian decomposition and a Pfaffian analogue of q-Catalan Hankel determinants, arxiv:1011.5941 (2011).

[6] Q.-H. Hou and Y.-P. Mu, Recurrent sequences and Schur functions, Adv. in Appl. Math. 31 (2003), no. 1, 150-162.

[7] D. Kazhdan and G. Lusztig, Representations of Coxeter groups and Hecke algebras, Invent. Math. 53 (1979), no. 2, 165-184.

[8] A. Kirillov and A. Lascoux, Factorization of Kazhdan-Lusztig elements for Grassmanians, Combinatorial methods in representation theory (Kyoto, 1998), 143-154, Adv. Stud. Pure Math., 28, Kinokuniya, Tokyo, 2000.

[9] A. Lascoux and M. P. Schützenberger, Polynômes de Kazhdan-Lusztig pour les grassmanniennes, in Tableaux de Young et Foncteurs de Schur, Torun 1980, Astérique 87-88 (1981).

[10] A. Lascoux, Symmetric Functions and Combinatorial Operators on Polynomials, CBMS/AMS Lecture Notes 99, 2003.

[11] _ Pfaffians and representations of the symmetric group, Acta Math. Sin. (Engl. Ser.) 25 (2009), no. 12, 1929-1950.

[12] A. Lascoux and P. Pragacz, Bezoutians, Euclidean algorithm, and orthogonal polynomials, Ann. Comb. 9 (2005), no. 3, 301-319.

[13] J.-G. Luque, Macdonald polynomials at $t=q^{k}$, J. Algebra 324 (2010), no. 1, 36-50.

[14] D. E. Rutherford, Substitutional Analysis, Edinburgh, at the University Press, 1948.

[15] Susan Y. J. Wu and Arthur L. B. Yang, Division and the Giambelli identity, Linear Algebra Appl. 406 (2005), 301-309.

[16] A. Young, The Collected Papers of Alfred Young, University of Toronto Press, 1977.

CNRS, IGM, Université de PARIS-Est 77454 Marne-La-Vallée CEDEX 2, UK

E-mail address: Alain.Lascoux@univ-mlv.fr 